TAUP-2081-93

\title{
Galilean Limit of Equilibrium Relativistic Mass Distribution
}

\author{
L. Burakovsky* and L.P. Horwitz ${ }^{\dagger}$ \\ School of Physics and Astronomy \\ Raymond and Beverly Sackler Faculty of Exact Sciences \\ Tel-Aviv University, Tel-Aviv 69978, ISRAEL
}

\begin{abstract}
The low-temperature form of the equilibrium relativistic mass distribution is subject to the Galilean limit by taking $c \rightarrow \infty$. In this limit the relativistic Maxwell-Boltzmann distribution passes to the usual nonrelativistic form and the Dulong-Petit law is recovered.
\end{abstract}

Key words: special relativity, relativistic Maxwell-Boltzmann, mass distribution, Galilean limit.

PACS: 03.30.+p, 05.20.Gg, 05.30.Ch, 98.20.-d

\section{Introduction}

In a previous paper [1] we studied an equilibrium relativistic ensemble, described by an equilibrium relativistic Maxwell-Boltzmann distribution with variable mass.

*Bitnet:BURAKOV@TAUNIVM.

${ }^{\dagger}$ Bitnet:HORWITZ@TAUNIVM. Also at Department of Physics, Bar-Ilan University, RamatGan, Israel 
For such a system a well-defined mass distribution was found, consistent in lowtemperature limit with the one obtained by Hakim [2] from the well-known JüttnerSynge distribution [3] of an on-mass-shell relativistic kinetic theory. Calculations of the average values of mass and energy gave in the low-temperature limit a correction of the order of $10 \%$ to the Dulong-Petit law.

In the present paper we consider the Galilean limit of low-temperature form of the equilibrium relativistic mass distribution. We show that no correction to the Dulong-Petit law appears in this limit of the theory.

\section{Preliminary remarks}

In a previous paper, having begun with the low-temperature form of the relativistic Maxwell-Boltzmann distribution $[1,(47)]$ (we use the metric $g^{\mu \nu}=(-,+,+,+), \quad q \equiv$ $q^{\mu}, \quad p \equiv p^{\mu}$, and take $\hbar=c=1$ unless otherwise specified)

$$
f(p, q)=C(q) e^{-A m_{c}^{2}} e^{2 A p_{\mu} p_{c}^{\mu}}
$$

which coincides with the Jüttner-Synge distribution adopted for an on-mass-shell relativistic kinetic theory, we obtained the following low-temperature form of the equilibrium relativistic mass distribution $[1,(48)]$ :

$$
f(m)=\frac{\left(2 A m_{c}\right)^{3}}{2} m^{2} K_{1}\left(2 A m_{c} m\right)
$$

where $m_{c}=\sqrt{-p_{c}^{\mu} p_{c \mu}}$ and $K_{1}$ is the Bessel function of the third kind (imaginary argument),

$$
K_{\nu}(z)=\frac{\pi i}{2} e^{\pi i \nu / 2} H_{\nu}^{(1)}(i z)
$$

In this formula $2 A m_{c}$ corresponds to $1 / k_{B} T[1,(13)]$.

The distributions (1),(2) gives the following values of the average four-momentum and mass:

$$
\left\langle p^{\mu}\right\rangle=4 \frac{p_{c}^{\mu}}{m_{c}} k_{B} T,\langle m\rangle=\frac{3 \pi}{4} k_{B} T .
$$

In the local rest frame we have

$$
\langle E\rangle=4 k_{B} T
$$

and, consequently,

$$
\langle E\rangle-\langle m\rangle=\gamma \frac{3}{2} k_{B} T
$$

where $\gamma=\frac{16-3 \pi}{6} \approx 1.1$ represents a relativistic correction to the Dulong-Petit law.

This result follows directly from equilibrium thermodynamics without imposing the geometrical restriction of the precise Galilean group to an infinitely sharp mass 
shell. The reason for appearance of such a correction is determined by the fact that the difference $E-m$, even in the low-temperature limit, does not correspond to the expression for nonrelativistic energy $\frac{\mathbf{p}^{2}}{2 m}$. Therefore, the result that we have found can not be considered as a nonrelativistic limit; it is actually a relativistic low-temperature limit alone.

As is well known [4], the structure of the Galilean group, the symmetry of nonrelativistic system, implies that the mass of a particle must be a constant intrinsic property.

In this paper we shall consider the Galilean limit of equilibrium relativistic ensemble which has been treated in the series of papers [1], [5], by taking $c \rightarrow \infty$ [6], [7]. We shall see that in the Galilean limit the difference $E-m$ approaches the nonrelativistic expression $\frac{\mathbf{p}^{2}}{2 M}$, where Galilean mass $M$ coincides with the particle's intrinsic parameter. This variable $\frac{\mathbf{p}^{2}}{2 M}$ turns out to be distributed over ensemble with the usual nonrelativistic Maxwell-Boltzmann distribution, due to the fact that the relativistic relation between the energy $E$ and the mass $m E^{2}=m^{2}+\mathbf{p}^{2}$ transforms in the Galilean limit to $E=m+\frac{\mathbf{p}^{2}}{2 M}$, giving rise to the Maxwell-Boltzmann distribution of the latter. The first moment of this distribution $\left\langle\frac{\mathbf{p}^{2}}{2 M}\right\rangle$ (which coincides with $\langle E-m\rangle$ ), takes the value $\frac{3}{2} k_{B} T$, in agreement with the Dulong-Petit law.

We recognize, however, that the applicability of the Galilean group is an idealization of a world which seems to be more correctly described by the Poincaré group, and the Galilean limit is just a reasonable approximation for the relativistic relation $E^{2}=m^{2}+\mathbf{p}^{2}$.

\section{Galilean limit of a free relativistic $N$-particle system}

We consider a system of $N$ particles in the framework of a manifestly covariant mechanics [8], both for the classical theory and for the corresponding relativistic quantum theory. For the classical case, the dynamical evolution of such a system is governed by the equations of motion that are of the form of Hamilton equations for the motion of $N$ events which generate the space-time trajectories (particle world lines). These events are considered as the fundamental dynamical objects of the theory; they are characterized by their positions $q^{\mu}=(c t, \mathbf{r})$ and energy-momenta $p^{\mu}=(E / c, \mathbf{p})$ in an $8 N$-dimentional phase space. The motion is parametrized by an invariant parameter $\tau$ [8], called the "historical time". The collection of events (called "concatenation" [9]) along each world line corresponds to a particle, and hence the evolution of the state of the $N$-event system describes, a posteriori, the history in space and time of an $N$-particle system.

For the quantum case the dynamical evolution is governed by a generalized Schrödinger equation for the wave function $\psi_{\tau}\left(q_{1}, q_{2}, \ldots, q_{N}\right) \in L^{2}\left(R^{4 N}\right)$, the Hilbert space of 
square integrable functions with measure $d q_{1} d q_{2} \cdots d q_{N} \equiv d^{4 N} q$, describing the distribution of events and representing the probability amplitudes for finding events at space-time points $\left(q_{1}^{\mu}, q_{2}^{\mu}, \ldots, q_{N}^{\mu}\right)$ at any instant $\tau$ :

$$
i \hbar \frac{\partial \psi_{\tau}\left(q_{1}, \ldots, q_{N}\right)}{\partial \tau}=K \psi_{\tau}\left(q_{1}, \ldots, q_{N}\right)
$$

where $K$ is the dynamical evolution operator (generalized Hamiltonian), of the same form for both classical and quantum cases.

We shall consider here a many-particle system, within the framework of the relativistic generalization of the usual nonrelativistic Boltzmann theory [5].

To study the nonrelativistic limit of a dilute gas of events, it is sufficient to treat the simplest case of a system of $N$ free particles with the Hamiltonian

$$
K_{0}=\sum_{i=1}^{N} \frac{p_{i \mu} p_{i}^{\mu}}{2 M_{i}}
$$

where $M_{i}$ are positive parameters, the given intrinsic properties of the particles, having the dimension of mass.

The Hamilton equations

$$
\frac{d q_{i}^{\mu}}{d \tau}=\frac{\partial K}{\partial p_{i \mu}}, \quad \frac{d p_{i}^{\mu}}{d \tau}=-\frac{\partial K}{\partial q_{i \mu}}, \quad i=1,2, \ldots, N
$$

yield, in this case,

$$
\frac{d q_{i}^{\mu}}{d \tau}=\frac{p_{i}^{\mu}}{M_{i}}, \quad \frac{d p_{i}^{\mu}}{d \tau}=0, \quad i=1,2, \ldots, N
$$

The evolution of the wave function is described by the equation

$$
i \hbar \frac{\partial \psi_{\tau}\left(q_{1}, \ldots, q_{N}\right)}{\partial \tau}=K_{0} \psi_{\tau}\left(q_{1}, \ldots, q_{N}\right)
$$

The wave function can be expressed as a Fourier transform

$$
\begin{aligned}
\psi_{\tau}\left(q_{1}, \ldots, q_{N}\right) & =\frac{1}{(2 \pi \hbar)^{4 N}} \int d^{4} p_{1} \cdots d^{4} p_{N} e^{\frac{i}{\hbar} \sum_{k=1}^{N} p_{k}^{\mu} q_{k \mu}} \psi_{\tau}\left(p_{1}, \ldots, p_{N}\right) \\
& =\frac{1}{(2 \pi \hbar)^{4 N}} \int d^{4} p_{1} \cdots d^{4} p_{N} e^{\frac{i}{\hbar} \sum_{k=1}^{N} p_{k}^{\mu} q_{k \mu}} e^{-\frac{i}{\hbar} K_{0} \tau} \psi_{0}\left(p_{1}, \ldots, p_{N}\right) \\
& =\frac{1}{(2 \pi \hbar)^{4 N}} \int d^{4} p_{1} \cdots d^{4} p_{N} e^{\frac{i}{\hbar} \sum_{k=1}^{N}\left(p_{k}^{\mu} q_{k \mu}-\frac{p_{k}^{\mu} p_{k \mu}}{2 M_{k}} \tau\right)} \psi_{0}\left(p_{1}, \ldots, p_{N}\right) .
\end{aligned}
$$

If this wave function is to be associated with particles, the function $\psi_{\tau}\left(p_{1}, \ldots, p_{N}\right)=$ $e^{-i / \hbar K_{0} \tau} \psi_{0}\left(p_{1}, \ldots, p_{N}\right)$ must have support in momentum space in a region which is in 
the neighborhood of definite masses (as pointed out in [7], these considerations are valid also in the presence of interaction, if it is not too strong). In the nonrelativistic limit this support should approach the corresponding definite mass shells, consistent with a representation of the Galilean group.

We shall, therefore, require that the quantities

$$
\epsilon_{i}=E_{i}-M_{i} c^{2}, \quad i=1, \ldots, N
$$

constructed of variables occuring in the integrand of (8), i.e., in the support of $\psi_{\tau}\left(p_{1}, \ldots, p_{N}\right)$, be finite as $c \rightarrow \infty$ (compared to all other velocities) for the states with finite momenta.

We shall see that it is sufficient that the support of $\psi_{\tau}\left(p_{1}, \ldots, p_{N}\right)$ contract such that the variables

$$
\eta_{i}=c^{2}\left(m_{i}-M_{i}\right), \quad i=1, \ldots, N
$$

may take any value, however, finite, as $c \rightarrow \infty$; or, equivalently,

$$
m_{i}=M_{i}\left(1+O\left(\frac{1}{c^{2}}\right)\right)
$$

(The situation is quite similar to one in relativistic classical statistical mechanics, when this freedom permits one to obtain the Galilean microcanonical ensemble [6].)

Indeed, in this case one can show that the values $E_{i}-m_{i} c^{2}$ are equal to

$$
E_{i}-m_{i} c^{2}=\frac{\mathbf{p}_{i}^{2}}{2 M_{i}}+O\left(\frac{1}{c^{2}}\right), \quad i=1, \ldots, N
$$

and approach nonrelativistic kinetic energies of particles with the Galilean masses $M_{i}$ as $c \rightarrow \infty$ :

$$
\begin{aligned}
E_{i}-m_{i} c^{2} & =\sqrt{\left(m_{i} c^{2}\right)^{2}+\mathbf{p}_{i}^{2} c^{2}}-m_{i} c^{2} \\
& =\sqrt{\left(\eta_{i}+M_{i} c^{2}\right)^{2}+\mathbf{p}_{i}^{2} c^{2}}-m_{i} c^{2} \\
& =M_{i} c^{2} \sqrt{1+\frac{2 \eta_{i}}{M_{i} c^{2}}+\frac{\eta_{i}^{2}}{M_{i}^{2} c^{4}}+\frac{\mathbf{p}_{i}^{2}}{M_{i}^{2} c^{2}}-m_{i} c^{2}} \\
& =M_{i} c^{2}\left(1+\frac{\eta_{i}}{M_{i} c^{2}}+\frac{\mathbf{p}_{i}^{2}}{2 M_{i}^{2} c^{2}}+O\left(\frac{1}{c^{4}}\right)\right)-m_{i} c^{2} \\
& =\left(M_{i} c^{2}+\eta_{i}\right)+\frac{\mathbf{p}_{i}^{2}}{2 M_{i}}-m_{i} c^{2}+O\left(\frac{1}{c^{2}}\right) \\
& =\frac{\mathbf{p}_{i}^{2}}{2 M_{i}}+O\left(\frac{1}{c^{2}}\right) .
\end{aligned}
$$

Consequently, the quantities

$$
\epsilon_{i}=E_{i}-M_{i} c^{2}=E_{i}-m_{i} c^{2}+\left(m_{i}-M_{i}\right) c^{2}=\frac{\mathbf{p}_{i}^{2}}{2 M_{i}}+\eta_{i}+O\left(\frac{1}{c^{2}}\right), \quad i=1, \ldots, N,
$$


are finite as $c \rightarrow \infty$, as was required from the very beginning.

Now we turn to investigate the behavior of the wave function $\psi_{\tau}\left(q_{1}, \ldots, q_{N}\right)$ in the Galilean limit:

$$
\begin{aligned}
\psi_{\tau}\left(q_{1}, \ldots, q_{N}\right)= & \frac{1}{(2 \pi \hbar)^{4 N}} \int d^{4} p_{1} \cdots d^{4} p_{N} e^{\frac{i}{\hbar} \sum_{k=1}^{N}\left(\mathbf{p}_{k} \mathbf{r}_{k}-E_{k} t_{k}\right)} e^{\frac{i}{\hbar} \sum_{k=1}^{N} \frac{m_{k}^{2} c^{2}}{2 M_{k}} \tau} \psi_{0}\left(p_{1}, \ldots, p_{N}\right) \\
= & \frac{1}{(2 \pi \hbar)^{4 N}} \int \frac{d E_{1}}{c} \cdots \frac{d E_{N}}{c} d^{3} \mathbf{p}_{1} \cdots d^{3} \mathbf{p}_{N} e^{\frac{i}{\hbar} \sum_{k=1}^{N}\left[\mathbf{p}_{k} \mathbf{r}_{k}-\left(\frac{\mathbf{p}_{k}^{2}}{2 M_{k}}+M_{k} c^{2}+\eta_{k}\right) t_{k}\right]} \\
& \times e^{\frac{i}{\hbar} \sum_{k=1}^{N}\left(\frac{M_{k} c^{2}}{2}+\eta_{k}\right) \tau} \psi_{0}\left(p_{1}, \ldots, p_{N}\right) \\
= & \frac{1}{(2 \pi \hbar)^{4 N}} \int \frac{d \eta_{1}}{c} \cdots \frac{d \eta_{N}}{c} d^{3} \mathbf{p}_{1} \cdots d^{3} \mathbf{p}_{N} e^{\frac{i}{\hbar} \sum_{k=1}^{N}\left(\mathbf{p}_{k} \mathbf{r}_{k}-\frac{\mathbf{p}_{k}^{2}}{2 M_{k}} t_{k}\right)} e^{-\frac{i}{\hbar} \sum_{k=1}^{N} M_{k} c^{2} t_{k}} \\
& \times e^{\frac{i}{\hbar} \sum_{k=1}^{N} \frac{M_{k} c^{2}}{2} \tau} e^{\frac{i}{\hbar} \sum_{k=1}^{N}\left(\tau-t_{k}\right) \eta_{k}} \psi_{0}\left(p_{1}, \ldots, p_{N}\right) .
\end{aligned}
$$

Although the support of $\psi_{0}\left(p_{1}, \ldots, p_{N}\right)$ is bounded in the $\eta_{k}$ 's as $c \rightarrow \infty$, the integrals over the $\eta_{k}$ 's can approximately yield factors of $\delta\left(t_{k}-\tau\right)$, as remarked in [7]. Consider the case for which $\psi_{0}\left(p_{1}, \ldots, p_{N}\right)$ is independent of $\eta_{k}$, for $k=1, \ldots, N$, in $-\triangle_{k} \leq$ $\eta_{k} \leq \triangle_{k}$ and is zero outside this region; then the wave function (15) is proportional to the product

$$
\begin{aligned}
\prod_{k=1}^{N} \int_{-\triangle}^{\triangle} d \eta_{k} e^{\frac{i}{\hbar} \eta_{k}\left(\tau-t_{k}\right)} & =\prod_{k=1}^{N} \int_{-\triangle / \hbar}^{\triangle / \hbar} \hbar d \eta_{k}^{\prime} e^{i \eta_{k}^{\prime}\left(\tau-t_{k}\right)} \\
& =(2 \pi \hbar)^{N} \prod_{k=1}^{N} \delta_{\triangle / \hbar}\left(\tau-t_{k}\right)
\end{aligned}
$$

where

$$
\triangle=\min \left(\triangle_{1}, \triangle_{2}, \ldots, \triangle_{N}\right)
$$

and

$$
\delta_{\triangle / \hbar}\left(\tau-t_{k}\right) \rightarrow \delta\left(\tau-t_{k}\right),
$$

iff $\hbar \rightarrow 0$ (it is clear now that $\triangle \rightarrow 0$ precisely would be an unsuitable condition for the nonrelativistic limit). The dispersion of $t_{k}$ around $\tau$, bounded by

$$
\left|t_{k}-\tau\right| \leq \hbar / \triangle_{k} \leq \hbar / \triangle
$$

is therefore a purely quantum effect (it does not depend on $c$ and vanishes with $\hbar \rightarrow 0$ ), emerging asymptotically from a relativistic quantum theory in the Galilean limit, as emphasised in [7].

\footnotetext{
${ }^{1} \triangle \rightarrow \infty$ may also satisfy this condition; in the present paper we shall use the fact that $\triangle$ can take any infinitesimal value but not zero.
} 
Thus we will have

$$
\int \frac{d \eta_{1}}{c} \cdots \frac{d \eta_{N}}{c} e^{\frac{i}{\hbar} \sum_{k=1}^{N}\left(\tau-t_{k}\right) \eta_{k}} \cong(2 \pi \hbar)^{N} \prod_{k=1}^{N} \delta\left(c \tau-c t_{k}\right),
$$

which means that the times associated with all of the particles become synchronized in the Galilean limit:

$$
c t_{1}=c t_{2}=\ldots=c t_{N}=c t=c \tau .
$$

This result also can be obtained from the canonical equations of motion:

we have with the help of (9)

$$
\begin{aligned}
K_{0}=\sum_{k=1}^{N} \frac{-E_{k}^{2} / c^{2}+\mathbf{p}_{k}^{2}}{2 M_{k}} & =\sum_{k=1}^{N}\left\{-\frac{1}{2 M_{k} c^{2}} \epsilon_{k}\left(\epsilon_{k}+2 M_{k} c^{2}\right)-\frac{M_{k} c^{2}}{2}+\frac{\mathbf{p}_{k}^{2}}{2 M_{k}}\right\} \\
& =\sum_{k=1}^{N} \frac{\mathbf{p}_{k}^{2}}{2 M_{k}}-\sum_{k=1}^{N} \epsilon_{k}-\frac{c^{2}}{2} \sum_{k=1}^{N} M_{k}-\sum_{k=1}^{N} \frac{\epsilon_{k}^{2}}{2 M_{k} c^{2}} .
\end{aligned}
$$

Since, according to the equations of motion,

$$
\frac{d}{d \tau} c t_{k}=-\frac{\partial K_{0}}{\partial\left(E_{k} / c\right)}=-\frac{\partial K_{0}}{\partial\left(\epsilon_{k} / c\right)}=c+\frac{\epsilon_{k}}{M_{k} c}
$$

it follows that

$$
c t_{k}=c \tau+\int_{0}^{\tau} \frac{\epsilon_{k}\left(\tau^{\prime}\right)}{M_{k} c} d \tau^{\prime}+c t_{k}(0)
$$

choosing now in the initial instant $t_{k}(0)=t(0)$ for all of the particles, $k=1, \ldots, N$, and taking $c \rightarrow \infty$, we obtain (18).

Finally, taking into account all of the abovementioned considerations, we can see that the initial wave function $\psi_{\tau}\left(q_{1}, \ldots, q_{N}\right)$ in the Galilean limit approaches the nonrelativistic expression

$$
\psi_{\tau}\left(\mathbf{r}_{1}, \ldots, \mathbf{r}_{N}, t\right)=\left(\frac{1}{2 \pi \hbar}\right)^{3 N} \int d^{3} \mathbf{p}_{1} \cdots d^{3} \mathbf{p}_{N} e^{\frac{i}{\hbar} \sum_{k=1}^{N}\left(\mathbf{p}_{k} \mathbf{r}_{k}-\frac{\mathbf{p}_{k}^{2}}{2 M_{k}} t\right)} e^{-\frac{i}{\hbar} \varphi} \psi_{0}\left(\mathbf{p}_{1}, \ldots, \mathbf{p}_{N}\right),
$$

up to an additional phase factor $e^{-\frac{i}{\hbar} \varphi}$, where

$$
\varphi=\frac{M c^{2} t}{2}, \quad M=\sum_{k=1}^{N} M_{k}
$$

\section{Galilean limit of the relativistic Maxwell-Boltzmann distribution}

We now wish to consider the Galilean limit of the equilibrium relativistic MaxwellBoltzmann distribution used in ref. [1],

$$
f_{0}(q, p)=C(q) e^{A\left(p+p_{c}\right)^{2}}
$$


which is normalized as follows,

$$
\int d^{4} p f_{0}(q, p)=n(q)
$$

where $n(q)$ is the total number of events per unit space-time volume in the neighborhood of the point $q$.

Since in the framework of the relativistic Boltzmann theory [1],[5] particles are considered as having equal intrinsic parameters,

$$
M_{1}=M_{2}=\ldots=M_{N}=M
$$

It then follows from the relations (we suppress $c$ for the present consideration)

$$
\eta=m-M, \quad-\triangle \leq \eta \leq \triangle
$$

that

$$
M-\triangle \leq m \leq M+\triangle .
$$

Since $\triangle$ may take any infinitesimal value ${ }^{2}$ but not zero, i.e., the variation in mass of the particles of the ensemble may be very small, we can take the value of $p^{2} \equiv-m^{2}$ restricted to a small neighborhood of a fixed value $-M^{2}$. This permits us to write (21) as

$$
f_{0}(q, p) \cong C(q) e^{-A\left(M^{2}+m_{c}^{2}\right)} e^{2 A p^{\mu} p_{c \mu}} .
$$

Introducing hyperbolic variables [5] and performing integration [10], we obtain from (22) and (24) the normalization relation

$$
n(q)=C(q) \frac{4 \pi \triangle M^{2}}{A m_{c}} e^{-A\left(M^{2}+m_{c}^{2}\right)} K_{1}\left(2 A M m_{c}\right),
$$

where $K_{1}$ is the Bessel function of the third kind (3).

Identifying Synge's Lagrange parameters [3],

$$
\xi^{\mu}=2 A p_{c}^{\mu}
$$

and hence $\xi=\sqrt{-\xi^{\mu} \xi_{\mu}}=2 A m_{c}$, we see that (24) coincides with the result of Synge [3] obtained directly from an on-mass-shell relativistic kinetic theory.

The average value of $p^{\mu}$ can be obtained, similar to [1], using the parametric differentiation with respect to $p_{c}^{\mu}$ :

$$
\left\langle p^{\mu}\right\rangle_{q}=p_{c}^{\mu} \frac{M}{m_{c}} \frac{K_{2}\left(2 A M m_{c}\right)}{K_{1}\left(2 A M m_{c}\right)}
$$

\footnotetext{
${ }^{2}$ It corresponds to infinitely sharp mass shell $\triangle=|m-M|$.
} 
The absolute temperature is defined, similar to [1],[5], through the relation

$$
2 A m_{c}=\frac{1}{k_{B} T}
$$

which implies that in thermal equilibrium $A m_{c}$ is independent of $q$; so that

$$
\left\langle p^{\mu}\right\rangle=p_{c}^{\mu} \frac{M}{m_{c}} \frac{K_{2}\left(M / k_{M} T\right)}{K_{1}\left(M / k_{B} T\right)} .
$$

As in [1],[5], to obtain the local energy density we make a Lorentz transformation to the rest frame of the local average motion. According to (29), the relative velocity of the new frame is

$$
\mathbf{u}=\frac{\mathbf{p}_{\mathbf{c}}}{m_{c}}
$$

The rest frame energy is then

$$
\left\langle E^{\prime}\right\rangle=\frac{\langle E\rangle-\mathbf{u} \cdot \mathbf{p}}{\sqrt{1-\mathbf{u}^{2}}}
$$

so that

$$
\left\langle E^{\prime}\right\rangle=M \frac{K_{2}\left(M / k_{B} T\right)}{K_{1}\left(M / k_{B} T\right)}
$$

Using the asymptotic formula $[11,(9.7 .2)]$

$$
K_{\nu}(z) \sim \sqrt{\frac{\pi}{2 z}} e^{-z}\left\{1+\frac{4 \nu^{2}-1}{8 z}+\cdots\right\}, \quad z \rightarrow \infty
$$

we obtain, for $T \rightarrow 0$,

$$
\left\langle E^{\prime}\right\rangle-M \cong \frac{3}{2} k_{B} T
$$

in agreement with the Dulong-Petit law. On the other hand, for $T \rightarrow \infty$, one may use another asymptotic formula [11,(9.6.9)]

$$
K_{\nu}(z) \sim \frac{1}{2} \Gamma(\nu)\left(\frac{z}{2}\right)^{-\nu}, \quad z \rightarrow 0,
$$

to obtain

$$
\left\langle E^{\prime}\right\rangle \cong 2 k_{B} T
$$

the result previously obtained in [1],[5],[6].

In conclusion we shall show that in the Galilean limit the variable $E-m=\frac{\mathbf{p}^{2}}{2 M}$ has the usual nonrelativistic Maxwell-Boltzmann distribution.

Since the normalization conditions for the low-temperature and the sharp-mass forms of the relativistic Maxwell-Boltzmann distribution (21) read

$$
n(q)=C(q) e^{-A m_{c}^{2}} \int d^{4} p e^{2 A p^{\mu} p_{c \mu}}
$$

\footnotetext{
${ }^{3}$ It follows from (1) and (24), respectively.
} 
and

$$
n(q)=C(q) e^{-A\left(M^{2}+m_{c}^{2}\right)} \int d^{4} p e^{2 A p^{\mu} p_{c \mu}},
$$

all the difference between these two forms is contained in the normalization factor. The remaining integral on $d^{4} p$ can be written in the local rest frame $p_{c}^{\mu}=\left(m_{c}, \mathbf{0}\right)$ $\left(2 A m_{c}=\frac{1}{k_{B} T}\right)$ :

$$
\int d E d^{3} \mathbf{p} e^{-\frac{E}{k_{B} T}}
$$

Taking into account (12), one can rewrite this expression as follows:

$$
\int d^{3} \mathbf{p} d m e^{-\left(m+\frac{\mathbf{p}^{2}}{2 M}\right) / k_{B} T}=\int d^{3} \mathbf{p} e^{-\frac{\mathbf{p}^{2}}{2 M}} \int d m e^{-\frac{m}{k_{B} T}} .
$$

The latter integral

$$
\int_{M-\triangle}^{M+\triangle} d m e^{-\frac{m}{k_{B} T}}=2 k_{B} T e^{-\frac{M}{k_{B} T}} \sinh \frac{\triangle}{k_{B} T}
$$

does not vanish since $\triangle$ is finite (it enters the normalization factor). We see that the freedom of $\triangle$ to take any value (in this case not necessary infinitesimal but $\leq M$ ), finite as $c \rightarrow \infty$ but not equal to zero, enables one to obtain the nonrelativistic Maxwell-Boltzmann distribution for $e \equiv \frac{\mathbf{p}^{2}}{2 M}$.

Finally, we have for the low-temperature form $\left.\right|^{\prime}$ of the relativistic Maxwell-Boltzmann distribution (21),

$$
n(q)=2 C(q) k_{B} T \sinh \frac{\triangle}{k_{B} T} e^{-\frac{m_{c}}{2 k_{B} T}} e^{-\frac{M}{k_{B} T}} \int d^{3} \mathbf{p} e^{-\frac{\mathbf{p}^{2}}{2 M}},
$$

which is the usual (normalized) nonrelativistic Maxwell-Boltzmann distribution

$$
f(e)=\frac{1}{\Gamma\left(\frac{3}{2}\right)\left(k_{B} T\right)^{3 / 2}} e^{1 / 2} \exp \left(-\frac{e}{k_{B} T}\right) .
$$

\section{Concluding remarks}

We have considered the Galilean limit of equilibrium relativistic ensemble. We have found that the relativistic relation between the energy $E$ and the mass $m$ transforms in this limit to $E=m+\frac{\mathbf{p}^{2}}{2 M}$, giving rise to the nonrelativistic Maxwell-Boltzmann distribution of $\frac{\mathbf{p}^{2}}{2 M}$. The first moment of this distribution $\left\langle\frac{\mathbf{p}^{2}}{2 M}\right\rangle$ (which coincides with $\langle E-m\rangle)$ is equal to $\frac{3}{2} k_{B} T$, in agreement with the Dulong-Petit law, and no relativistic correction appears in this limit, in contrast to [1].

\footnotetext{
${ }^{4}$ For the sharp-mass form there is an additional factor $e^{-A M^{2}}$ in the r.h.s. of (35).
} 
For the case of an equilibrium relativistic ensemble of indistinguishable events [12] the distribution function is found to be

$$
f(q, p)=C(q) \frac{1}{e^{-A\left(p+p_{c}\right)^{2}} \pm 1} ;
$$

in the Galilean limit it becomes the usual nonrelativistic distribution of Bose-Einstein or Fermi-Dirac, with chemical potential $\mu_{G}=\mu-M$, where $\mu$ is the chemical potential of relativistic theory [6] and $M$ is the Galilean mass.

\section{References}

[1] L. Burakovsky and L.P. Horwitz, Physica A, in press

[2] R. Hakim, J. Math. Phys. 15 (1974) 1310

[3] J.L. Synge, The Relativistic Gas, (North-Holland, Amsterdam, 1957)

[4] J.M. Levy-Leblond, J. Math. Phys. 4 (1963) 776

[5] L.P. Horwitz, S. Shashoua and W.C. Schieve, Physica A 161 (1989) 300

[6] L.P. Horwitz, W.C. Schieve and C. Piron, Ann. Phys. (N.Y.) 137 (1981) 306

[7] L.P. Horwitz and F.C. Rotbart, Phys. Rev. D 24 (1981) 2127

[8] L.P. Horwitz and C. Piron, Helv. Phys. Acta 46 (1973) 316

[9] R. Arshansky, L.P. Horwitz and Y. Lavie, Found. Phys. 13 (1983) 1167

[10] I.S. Gradshteyn and I.M. Ryzhik, Tables of Integrals, Series, and Products (Academic Press, New York, 1980) p.358, subsection 3.547, formula 9

[11] M. Abramowitz and I.A. Stegun, Handbook of Mathematical Functions, p.375, (Dover, New-York, 1970)

[12] L. Burakovsky and L.P. Horwitz, in preparation 